\title{
Natural Products: An Important Source for the Treatment of Psychosis
}

\author{
Shweta Kushwaha ${ }^{1}$ and Anupam Maurya ${ }^{2 *}$ \\ ${ }^{1}$ J.H.S., Jarauli Sherpur, Basic Education Board (U.P.), Hardoi, India \\ ${ }^{2}$ Pharmacopoeia Commission for Indian Medicine and Homoeopathy, Ministry of AYUSH, Ghaziabad, India
}

Received: 眥July 11, 2018; Published: 劃July 19, 2018

*Corresponding author: Dr. Anupam Maurya (Scientific Officer), Pharmacopoeia Commission for Indian Medicine and Homoeopathy, Ministry of AYUSH, Govt. of India, Ghaziabad, India 201002, Email: manupamk@gmail.com

\section{Introduction}

Psychosis is a mental disorders or psychiatric disorders due abnormal condition of the mind that results in difficulties telling what is real and what is not [1]. It is a wide range of condition that affects mood, behaviour and thinking. Psychosis is characterized by hallucination in vision, hearing, smelling and tasting etc. The person suffered from psychiatric disorders may include symptoms of false beliefs and seeing or hearing things that others do not see or hear [1]. Other symptoms may include disorganized thinking, incoherent speech and behaviour that are inappropriate for the situation [2]. There may also be, sleep problems, loss of motivation and social withdrawal, and difficulties carrying out daily activities [2]. The person experiencing psychosis may also have thoughts that are contrary to actual evidence; these thoughts are known as delusions. Psychosis is associated with several medical disorders and conditions like schizophrenia, schizophreniform disorder, and schizoaffective disorder: delusional disorder, substance-induced psychosis, dementia and bipolar disorder (manic depression) Figure 1[2,3].

People with psychosis may experience:
a) difficulty concentrating
b) depressed mood
c) sleeping too much or not enough
d) anxiety
e) suspiciousness
f) withdrawal from family and friends
g) delusions
h) hallucinations
i) disorganized speech, such as switching topics erratically

j) depression

k) suicidal thoughts or actions

Psychosis is one of the most dreaded disease of the $20^{\text {th }}$ century and spreading further with continuance and increasing incidences in $21^{\text {st }}$ century. In 2004, the World Health Organisation estimated that over 26 million people suffered from schizophrenia, making it one of the top 20 causes of disability worldwide[3]. Furthermore, in 2004, 30,000 deaths were attributed to the condition[3]. According to the 2013 Global Burden of Disease study, the predominant mental health problem worldwide is depression, followed by anxiety, schizophrenia and bipolar disorder. Globally, more than 300 million people suffer from depression, and 260 million suffer from anxiety disorders many of whom live with both conditions. A study by the World Health Organization found that such disorders cost the global economy $\$ 1$ trillion in lost productivity each year $[4,5]$. The treatment of psychosis depends on the specific diagnosis (such as schizophrenia, bipolar disorder or substance intoxication). The first-line treatment for many psychotic disorders is antipsychotic medication. Antipsychotics, also known as neuroleptics or major tranquilizers. They are also associated with increased mortality in elderly people with dementia. There are two types of Antipsychotic drugs First-generation antipsychotics, known as typical antipsychotics, were discovered in the 1950 s. Most second-generation drugs, known as atypical antipsychotic drugs. The long-term use of antipsychotics is associated with side effects such as involuntary movement disorders, gynecomastia, and metabolic syndrome $[2,4,5]$.

\section{Natural Products as a antipsychotic agents}

Natural antipsychotic agents (Natural Products) are an important area of the current research and are in good demand all over the world because they are better than synthetic drugs as 
they do not possess serious side effects and chronic toxicity [6]. Natural products are plant/animal derived secondary metabolites or chemical compounds possess enormous structural and chemical diversity that is unsurpassed by any synthetic libraries. Ancient traditional medicine systems in the world have revolved around use of rich heritage of extracts from plant sources and phytochemicals derived from them. There are various herbs present in Ayurveda like Bacopa monnieri, Glycerriza glabra, Acormus calmus, Withania somnifera, Carumrox burghianum, Cuminum cyminum, Carum carvi, Zingiber officinalis, Piper nigrum, Piper longum and Cissampe lospareira which are quit beneficial to manage the problem of phychsosis and support the good brain health [7]. In the present scenario the number of pharmaceutical products available based on plant products. A great number of health products dietary supplements and cosmetic products and the food products have adapted phytoextracts and phytochemicals as major active ingredients.

Indole compounds especially indole alkaloids from plant are historically constituted a class of major importance in the development of new plant derived drugs. In Nigeria, Alstonia and some other species have been traditionally used as major plant based component for the treatment of mental illness [6,8]. The indole alkaloid. alstonin (i) found in various plant species including Alstonia sps., Catharanthus roseus, Picralima nitida, Rauwolfia caffra and Rauwolfia vomitoria [1]. Alstonine is a putative antipsychotic constituent and its antipsychotic profile is remarkably coherent with its use by Nigerian traditional psychiatrists [8]. The genus Rauwolfia is best supported as an antipsychotic and in eastern medicine it also continues to be used as a tranquilizer. Main active constituents of this genus are indole alkaloids. The group of alkaloids got more attention after the isolation of an important indole alkaloids known as reserpine (ii) from Rauwolfia serpentina Benth. Resperine shows antipsychotic and antihypertensive activity and has been used for the control of high blood pressure and for the relief of psychotic symptoms [9]. Bioactvity guided extraction and isolation of Antipsychotic agents from the leaf of Rauwolfia tetraphylla were carried out by Srivastava et al. which afforded six indole alkaloids: 10-methoxytetrahydroalstonine (iii) , isoreserpiline (iv), an isomeric mixture of 11-demethoxyreserpiline (v) and 10-demethoxyreserpiline (vi), $\alpha$-yohimbine (vii) and reserpiline (viii), the chloroform extract and alkaloids (v-viii) showed significant antipsychotic activity in a dose dependent manner without any extra pyramidal symptoms or side effect at the tested doses [6,10].

Bacopa monnieri (Brahmi), an Ayurvedic herb, which is primarily used as memory enhancer and and neuropharmacological disorders like insomnia, insanity, depression, anxiety, psychosis, epilepsy and stress, the add on effect of Brahmi has been found in management of schizophrenia [11]. The pharmacological properties of Bacopa monnieri were attributed mainly due to the presence of characteristic saponins called as bacosides, in which bacosides A and B are major constituents in Bacopa monnieri. Bioactive bacosides A and B, bacopasides I and II and bacopasaponin $\mathrm{C}$ exhibited antidepressant activity when tested on experimental animals [12,13]. Withania somnifera (Ashwagandha) is important medicinal plant used in Traditional Indian System of Medicine. W. somnifera having many pharmacological activities such as anxiolytic, antidepressant, antimicrobial, antimalarial, cardioprotective and neuroprotective. The active ingredients of Withania somnifera are alkaloids, steroidal lactones (withanolides and withaferins) and saponins. Sitoindosides VII-X and Withaferin A are the active compounds of Withania, which showed significant antistress activity against acute models of experimental stress [14].

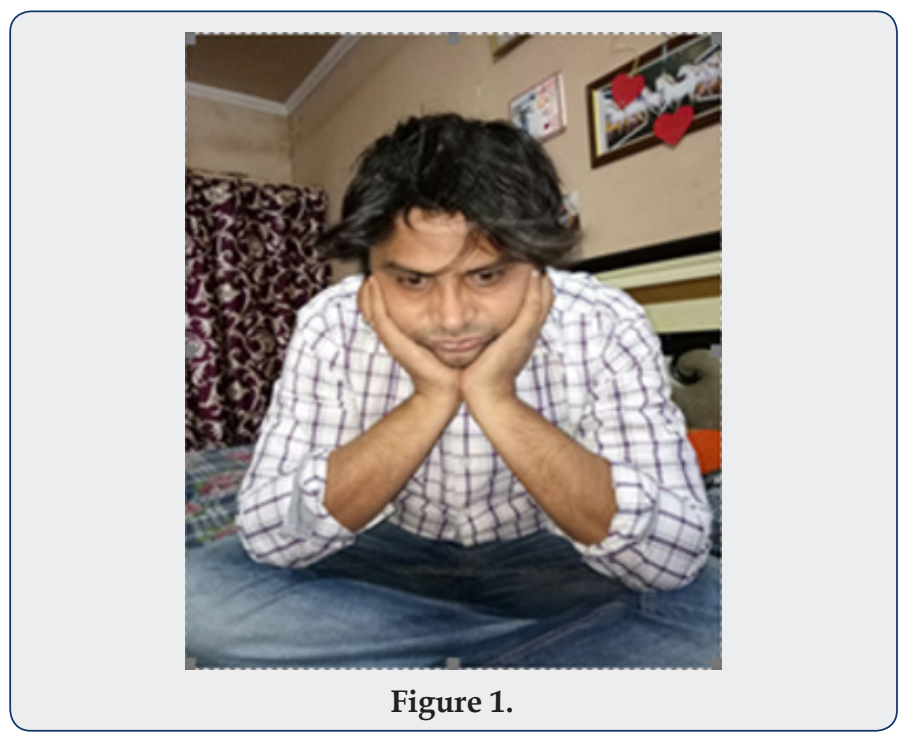

\section{Epilogue}

Many plant species have been using as a traditional medicine in word since very long time, this exert most powerful approach of drug discovery by systematic follow-up of ethno medical (traditional medicine) uses of plants. The traditional medicine mainly derived from plants source, have been prescribed for the various type of disease and disorder including psychosis due to their cost effective option and less side effects. Natural Product chemistry is a divergent area of research in drug discovery and development which associated with isolation, purification, characterization and identification of the pharmacological areas of the chemical product. Some of the plant derived molecules are useful as antipsychotic drugs as themselves while the others have been modified semisynthetically to produce greater effect as a drug. Most of the times suitable structure modifications have provided new leads or drugs, with enhanced activity and less toxicity. Recently, a suitable example of systematic activity guided extraction and isolation of antipsychotic compounds viz; an isomeric mixture of 11-demethoxyreserpiline (v) and 10-demethoxyreserpiline (vi), $\alpha$-yohimbine (vii) and reserpiline (viii) from the leaf of Rauwolfia tetraphylla have been studied. All the isolated compounds showed 
significant antipsychotic activities without any extra pyramidal symptoms or side effect. Hence, many plant species show good antipsychotic activity at plant and extracts level and traditionally using in the treatment of psychosis [15]. In order to find out more potent antipsychotic agents from plants, the main focus on activity guided extraction and isolation of chemical compounds and structure modification of isolates are required, which may be provided suitable leads for drug discovery and development of antipsychotic drugs from very common natural origin (Figure 2).

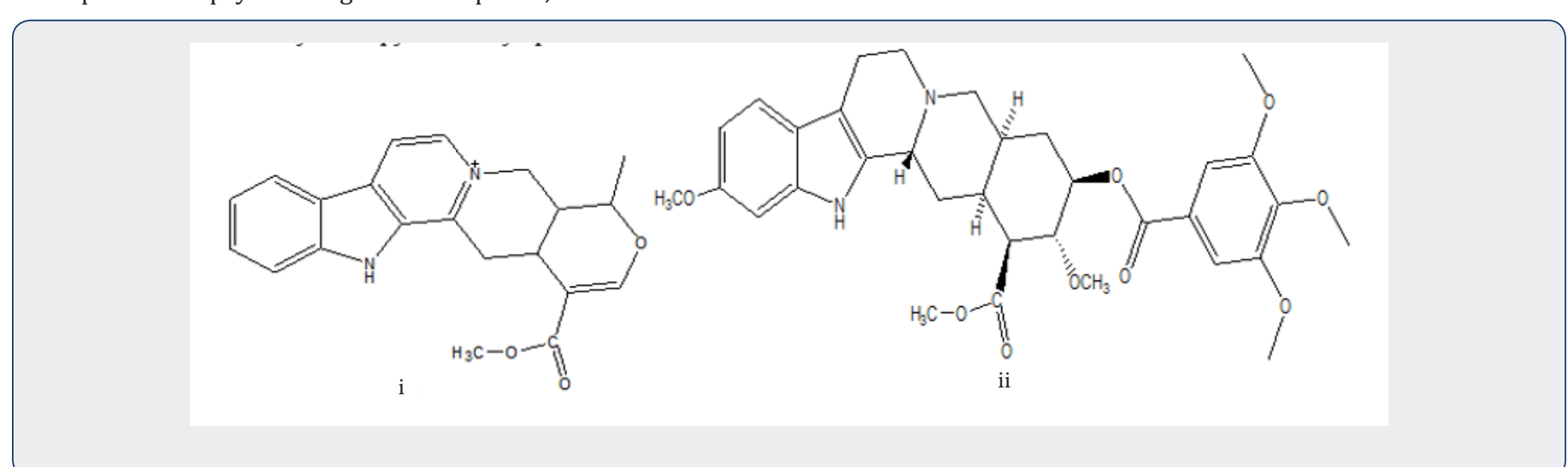

\section{Acknowledgements}

Authors are thankful to Pharmacopoeia Commission for Indian Medicine and Homeopathy (PCIM\&H), Ghaziabad and Basic Education Board, U.P. for providing necessary facilities to write this article.

\section{References}

1. (2018) RAISE Questions and Answers. NIMH.

2. Van Gogh's (1889) shows changes in light and color as can appear with psychosis. Psychosis.

3. (2019) Mental Health Day on the Hill-Issue Highlights p. 1-7.

4. Ayurvedic Treatment of psychosis. Planet Ayurveda.

5. (2010) mhGAP Evidence Resource Centre. Mental health.

6. (2016) Fundamental Facts About Mental Health. Mental Health Foundation, London.

7. Srivastava SK, Agrawal AK, Singh SC, Khanna VK, Singh J, et al. WO/2010/113180.
8. Ayurvedic Treatment of psychosis. Planet Ayurveda.

9. Elisabetsky E, Costa CL (2006) The Alkaloid Alstonine: A Review of Its Pharmacological Properties. Evid Based Complement Alternat Med 3(1): $39-48$.

10. Pant A, Pandey R (2015) Bioactive phytomolecules and ageing in Caenorhabditis elegans. Healthy Aging Research 4:19.

11. Gupta S, Khanna VK, Maurya A, Bawankule DU, Shukla RK et al. (2012) Bioactivity guided isolation of antipsychotic constituents from the leaves of Rauwolfia tetraphylla L. Fitoterapia 83(6): 1092-1099.

12. Sarkar S, Mishra BR, Praharaj SK, Nizamie SH (2012) Add-on effect of Brahmi in the management of schizophrenia. J Ayurveda Integr Med 3(4): 223-225.

13. Kishore K, Singh M (2005) Effect of bacosides, alcoholic extract of Bacopa monniera Linn. (brahmi), on experimental amnesia in mice Indian Journal of Experimental Biology 43: 640-645.

14. Al Snafi AE (2013) The pharmacology of Bacopa monniera. A review, International Journal of Pharma Sciences and Research (IJPSR) 4: 154159.

15. Verma KC (2010) Ashwagandha (Withania Somnifera DUNAL): WONDER Medicinal Plant. Agri. Review 31(4): 292-297.

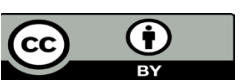

This work is licensed under Creative Commons Attribution 4.0 License

To Submit Your Article Click Here: Submit Article

DOI: 10.32474/AOICS.2018.03.000162

\section{AOICS}

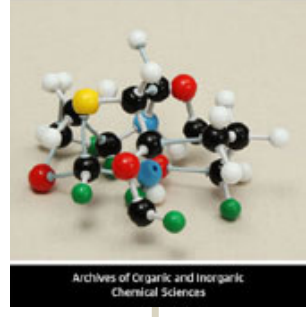

Archives of Organic and Inorganic Chemical Sciences

\section{Assets of Publishing with us}

- Global archiving of articles

- Immediate, unrestricted online access

- Rigorous Peer Review Process

- Authors Retain Copyrights

- Unique DOI for all articles 